Philosophie ANTIQUE
Philosophie antique

Problèmes, Renaissances, Usages

13 | 2013

Analogie et connaissance

\title{
Diego E. MACHUCA (éd.), New Essays on Ancient Pyrrhonism
}

\section{Lorenzo Corti}

\section{OpenEdition}

\section{Journals}

Édition électronique

URL : https://journals.openedition.org/philosant/919

DOI : 10.4000/philosant.919

ISSN : 2648-2789

\section{Éditeur}

Éditions Vrin

\section{Édition imprimée}

Date de publication : 1 novembre 2013

Pagination : $273-277$

ISBN : 978-2-7574-0591-8

ISSN : 1634-4561

Référence électronique

Lorenzo Corti, « Diego E. machuca (éd.), New Essays on Ancient Pyrrhonism », Philosophie antique [En ligne], 13 | 2013, mis en ligne le 01 novembre 2018, consulté le 03 décembre 2022. URL : http:// journals.openedition.org/philosant/919; DOI : https://doi.org/10.4000/philosant.919

\section{(ब) $\Theta \Theta$}

Creative Commons - Attribution - Pas d'Utilisation Commerciale - Pas de Modification 4.0 International - CC BY-NC-ND 4.0

https://creativecommons.org/licenses/by-nc-nd/4.0/ 
branche droite du lambda de sept nombres que Platon utilise pour structurer l'harmonie fondamentale de l'âme du monde.

Sans aller plus avant dans les détails du commentaire, c'est sans hésitation que nous reconnaissons la qualité soignée de cette publication très rapidement menée à terme et l'utilité indéniable de ce travail très richement documenté, fourni par un jeune chercheur tout à fait érudit, pour tous les connaisseurs - mathématiciens, musiciens, astronomes, philologues ou philosophes - de Théon de Smyrne.

Joëlle DeLATTRE

D. E. Machuca (éd.), New Essays on Ancient Pyrrhonism, Leyde/Boston, Brill (Philosophia Antiqua 126), 2011, XI + 207 p. ISBN 9789004207769.

Le volume offre huit articles sur le scepticisme pyrrhonien. Les essais, de la plume de M. Bonazzi, T. O'Keefe, J. Warren, F. Grgić, H. Thorsrud, S. Marchand, D. E. Machuca et O. Bueno, sont tous en anglais. Précédés d'une préface, d'une notice sur les auteurs et d'une brève introduction, ils sont suivis d'index des noms propres, des passages et des sujets. Chaque contribution est munie d'une bibliographic.

Les deux premiers articles sont consacrés à la section des Esquisses Pyrrboniennes (PH I, 209-241) où les différences entre pyrrhonisme et philosophies voisines sont discutées. En 221-225, Sextus soutient que la position de Platon n'est pas assimilable au pyrrhonisme - la remarque sextienne étant introduite, dans les

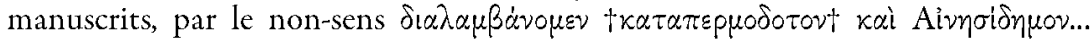
ótı... (222). Sextus soutient-il ceci en accord avec ses prédécesseurs pyrrhoniens Énésidème en particulier? La contribution de Bonazzi s'efforce de répondre à cette vexata quaestio par l'affirmative en quatre mouvements. Primo, il est judicieux d'accepter l'émendation au texte de Spinelli («Sextus Empiricus, the Neighbouring Philosophies and the Sceptical Tradition $\gg$ dans J. Sihvola [éd.], Ancient

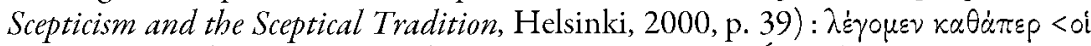

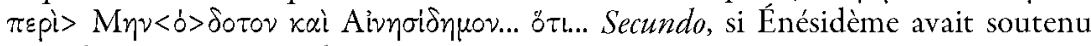
que Platon était un pyrrhonien, Sextus aurait contesté cette interprétation - ce qui n'est pas le cas. (Un mouvement à revoir ? Cet argumentum e silentio, discutable en soi, est affaibli par la prémisse douteuse selon laquelle les remarques de Sextus, en 222, ne veulent pas démontrer que Platon possédait des $\delta \dot{\delta} \gamma \mu \alpha \tau \alpha$.) Tertio, l'argument de Sextus contre l'interprétation sceptique de Platon en 222 trouve un parallèle dans ceux d'Énésidème contre le scepticisme académique (Photius, Bibliothéque, cod. 212, 170a31-38): Sextus et Énésidème semblent mobiliser des arguments semblables contre la même cible - l'Académic. Quarto, deux témoignages souvent utilisés pour montrer que certains pyrrhoniens considéraient Platon comme un collègue sceptique, l'un tiré d'un commentaire anonyme au Théétète de Platon, l'autre d'une introduction néoplatonicienne à sa philosophie, ne parlent pas des pyrrhoniens, mais des académiciens.

En $P H$ I, 215, Sextus rapporte la thèse selon laquelle cyrénaiques et sceptiques disent que nous apprenons seulement nos affects. Si la position cyrénaïque est bien attestée, sa contrepartie sceptique est problématique : Sextus ne la commente pas et en PH I, 200-201, la nie implicitement. Quelle est donc la différence entre les 
relations que cyniques et sceptiques entretiennent avec leurs affects et impressions? C'est la question à laquelle la contribution de O'Keefe est consacrée. L'auteur montre qu'en dépit de quelques assonances entre les caractérisations sceptique et cyrénaïque des affects/impressions, cette différence est remarquable. Les cyrénaïques utilisent des phrases comme « Je suis adouci », qui sont censées rapporter ce qui s'offre à l'expérience du sujet percevant, sans référence à des objets externes. Cette pratique est justifiée par leur projet de fournir une analyse des affects qui montre que seuls ceux-ci peuvent être connus, puisqu'ils sont intrinsèquement et irréversiblement évidents, de sorte que l'on peut faire des assertions infaillibles et irréfutables à leur égard.

Le sceptique à la Sextus, en revanche, décrit typiquement ses impressions par des phrases de la forme $\ll x$ me semble $F \gg$. Il s'agit là pour l'essenticl d'une façon de décrire comment un objet semble être, et pas seulement les états du sujet percevant. En rapportant comment l'objet lui apparaît, le sceptique n'est pas attaché à la thèse selon laquelle l'objet est comme il apparaît.

Le pyrrhonien n'a aucune théorie sur le contenu des ses affects et l'accès cognitif qu'on peut y avoirqui justifie sa pratique, car il n'a pas de théorie tout court. (L'article, qui tourne autour de la question de savoir si un sceptique apprend - $\kappa \alpha \tau \alpha \lambda \alpha \mu \beta \dot{\alpha} \nu \varepsilon$ - ses affects/impressions, aurait peut-être bénéficié d'une analyse de la distinction, faite par Sextus en $P H$ II, 1-10, entre sens épistémique et non épistémique de ce verbe : $c$. Corti, Scepticisme et langage, Paris, 2009, p. 188-206; Fine, «Sceptical Inquiry», dans D. Charles (éd.), Definition in Ancient Philosophy, Oxford, 2010, p. 493-525.

En $A M$ 162-166, Sextus propose un argument contre l'existence des dieux fondé sur l'idée que connaître la douleur implique de l'avoir ressentie. La contribution de Warren offre une riche analyse du passage. Elle inclut, du point de vue historique, une discussion des positions dogmatiques envisagées par Sextus ainsi que des différences entre son argument et un locus similis cicéronien - De Natura Deorum, III 38. Du point de vue philosophique, Warren se concentre sur la question de savoir si la défense sextienne de l'idée selon laquelle la connaissance de la douleur implique d'en avoir fait l'expérience anticipe la caractérisation moderne de la douleur comme une expérience essentiellement privée et subjective. Une analyse détaillée conduit l'auteur à répondre par la négative. Sextus exclut qu'il soit possible de connaitre la douleur de façon indirecte - sur la base de comptes-rendus des personnes qui la ressentent - non parce qu'il s'agit d'un état essentiellement privé, auquel le sujet qui l'éprouve a un accès cognitif privilégié, mais plutôt parce que ces rapports sont différents et incompatibles - ce qui laisse ouverte la possibilité que, s'ils ne l'étaient pas, il serait possible de connaître la douleur de façon indirecte.

En $P H$ I, 23, Sextus soutient que le sceptique vit «sans avoir de croyances en suivant l'observation de la vie quotidienne $\gg$. La contribution de Grgić vise à illustrer la notion de vie quotidienne en jeu dans le passage et sa compatibilité avec l'attitude sceptique. Une discussion détaillée de la thèse sextienne dans son contexte (qui aurait pu être éclaircie par une analyse de la condition qu'elle est supposée remplir en $P H$ I, 23-24 : expliquer les actes sceptiques) conduit l'auteur à en distinguer deux interprétations. Sextus pourrait suggérer que le sceptique approche 
sans croyance la vie quotidienne ou ordinaire, ou bien qu'une vie sans croyance est une vie ordinaire; mais dans le premier cas la distinction entre vie quotidienne et non quotidienne devient opaque, et dans le second on ne voit pas comment le sceptique, qui suspend son jugement sur des propositions acceptées dans la vie quotidienne, pourrait en être partisan. L'auteur sort le sceptique sextien de l'impasse en distinguant entre propositions ordinaires authentiques, utiles dans la vie quotidienne et utilisées de cette façon, et propositions ordinaires non authentiques, telles qu'elles sont utilisées dans les arguments philosophiques, et en suggérant que le domaine de la suspension sceptique n'inclut que les secondes.

D'après Sextus, le sceptique à la fois suspend son jugement sur l'existence des dieux, dit que les dieux existent et exercent leur providence, et accepte la pitié comme un bien. Il semble ainsi s'exposer à l'objection d'insincérité : avoir des croyances sur les dieux est nécessaire pour se conduire pieusement; le sceptique ne les a pas; done il ne peut pas se conduire ainsi - être sincèrement religieux. L'article de Thorsrud explore deux défenses du pyrrhonien. La première, inspirée par Annas, indique que le sceptique suspend son jugement sur les croyances théologiques, qui sont censées révéler des propriétés vraies d'objets non évidents et sont fondées sur des considérations rationnelles, mais possède les croyances religieuses qui n'ont pas ces caractéristiques et lui permettent d'agir pieusement. Cette stratégie est, selon Thorsrud, à rejeter, car les croyances religieuses telles

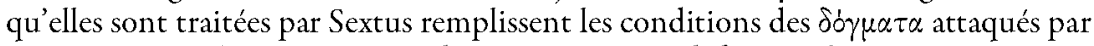
son sceptique. L'auteur propose donc une stratégie défensive alternative. Le noyau de l'objection anti-sceptique présuppose que le sceptique n'a pas les états affectifs associés à une pratique religieuse sincère, car ceux-ci sont causés par des croyances pertinentes. Thorsrud montre que ce présupposé est faux : au sceptique il peut sembler que les dieux exercent leur providence même après avoir suspendu son jugement à ce sujet; cette impression produira les états affectifs (peur, révérence...) qui fondent une pratique religieuse sincère.

Le sceptique ne sait rien ; comment donc peut-il écrire ? Pyrrhon n'écrivit rien ; mais les pyrrhoniens Timon et Sextus, eux, écrivirent. Comment ont-ils essayé de concilier scepticisme et écriture? La contribution de Marchand affronte cette question à travers une analyse du style d'écriture et des ouvrages de Timon et de Sextus. Si Timon essaye d'exposer la philosophie pyrrhonienne de façon non dogmatique en utilisant la poésie, l'humour et la parodie, Sextus le fait en introduisant un nouveau mode d'écriture, qui se manifeste dans sa caractérisation de l'usage sceptique du langage, sa stratégie d'écriture pragmatique et son usage de l'histoire de la philosophie. Le discours de Sextus exprime ce qui lui apparaît : il ne veut pas offrir une description objective de la réalité, mais le rapport d'une expérience subjective. Les expressions sceptiques ne sont censées impliquer la possession d'aucune croyance. Sextus écrit de façon pragmatique, ce qui implique de ne pas se battre sur les phrases, d'élucider le sens des termes utilisés, de renoncer à la précision, d'être concis. In fine, l'exigence de montrer qu'à chaque thèse dogmatique s'oppose une thèse égale conduit Sextus à écrire une histoire de la philosophie sui generis: une histoire du désaccord philosophique dogmatique qui devrait conduire au silence sceptique. 
En $P H$, Sextus caractérise son sceptique comme un individu qui suspend son jugement par rapport à tout objet d'enquête. En $A M \mathrm{XI}$, il semble lui attribuer les croyances selon lesquelles (i) rien n'est invariablement bon/mauvais, mais (ii) $x$ est bon/mauvais relativement aux personnes et aux circonstances. $P H$ et $M \mathrm{XI}$ sont-ils conciliables? L'essai de Machuca affronte cette question en considérant la réponse que Bett lui fournit et en en proposant une autre. Selon Bett, la partie de $A M \mathrm{XI}$ où Sextus attribue au sceptique les croyances (i) et (ii), qui inclut les sections 1-167, provient d'une source préexistante à celle d'où dérive la plus grande part du corpus sextien et présente un scepticisme de saveur énésidémienne. Une analyse détaillée des passages pertinents conduit l'auteur à soutenir contre Bett que, en dépit des apparences, le sceptique protagoniste de $A M \mathrm{XI}, 1-167$, ne croit pas que (ii). On ne peut pas nier que certains passages de $A M$ XI caractérisent le sceptique comme quelqu'un qui croit que (i), et que cette croyance est nécessaire pour atteindre la tranquillité. Mais ailleurs dans la même ouvre le sceptique est dit aussi suspendre son jugement universellement et atteindre la tranquillité via l'èno $\eta \dot{\eta}$. Machuca conclut que la coexistence des ces caractérisations en conflit est le signe du fait qu'en $A M \mathrm{XI}$ - pas seulement en $A M$ VII-X, comme Bett le suggère - Sextus essaye d'intégrer dans la variante du scepticisme qu'il adopte une version antérieure, sans toujours parvenir à le faire.

Dans son étude des cinq modes d'Agrippa, Barnes (The Toils of Scepticism, Cambridge, 1990) met en scène un dialogue entre un pyrrhonien et un dogmatique, où le premier essaie de montrer au second que, pour n'importe quelle proposition $P$, on n'est pas justifié à croire que $P$. À une étape de ce dialogue $B$ arnes puise chez Sextus, $A M$ VII, 52, une manœuvre à offrir au pyrrhonien. Selon Barnes, Sextus suppose ici que savoir que $P$ implique savoir savoir que $P$ - une thèse internaliste qui, jointe à d'autres, implique que le dogmatique n'est pas justifié à croire que $P$. La communication de Bueno veut critiquer l'interprétation de Barnes. Selon l'auteur, celle-ci suggère que, à l'étape mentionnée, le pyrrhonien accepte la thèse internaliste. Bueno indique que cette position est contradictoire, et propose une autre façon de comprendre $A M$ VII, 52, ainsi que l'attitude pyrrhonienne envers l'internalisme. $A M$ VII, 52, n'engage pas le pyrrhonien à l'internalisme. Il s'agit là d'une doctrine philosophique sur la nature de la connaissance; le pyrrhonien, après avoir considéré les arguments en sa faveur et ceux en faveur de la doctrine opposée, l'externalisme, ne peut que suspendre son jugement à leur égard. (Je dois avouer que la cible de cet article me semble fantomatique. Au cours de leur discussion des modes d'Agrippa, Sextus Empiricus et Jonathan Barnes font appel à des thèses de la théorie de la connaissance qui impliquent que le dogmatique n'est pas justifié de croire que $P$ et les prêtent au sceptique - en imaginant qu'il s'y réfère pour montrer au dogmatique qu'il n'est pas justifié à croire que $P$. Les deux font l'hypothèse que le sceptique peut le faire - que l'on peut expliquer le fait qu'il y fasse appel sans lui attribuer de croyance ; ni le savant grec ni le britannique ne présupposent la thèse, évidemment contradictoire, selon laquelle le sceptique doit accepter qu'elles sont vraies afin de le faire.)

Les essais que Machuca nous offre dans ce volume sont originaux, rafraîchissants et stimulants: les quelques questions que je leur ai adressées ne sont 
qu'un échantillon de celles que tout interprète du pyrrhonisme antique éprouverait du plaisir à discuter avec leurs auteurs.

Lorenzo CORTI

Porphyre, Sur la manière dont l'embryon reçoit l'âme. Études d'introduction, texte grec, traductions française et anglaise : travaux édités sous la responsabilité de L. Brisson avec la collaboration de G. Aubry, M.-H. Congourdeau et F. Hudry, Paris, Librairic philosophique J. Vrin, Paris, 2012 (Histoire des Doctrines de l'Antiquité Classique, 43), 384 p. ISBN 9782711624324.

Le Sur la manièe dont l'embryon recoit l'âme, souvent appelé Ad Gaurum du nom de son dédicataire Gauros, est un traité qui fut longtemps attribué au médecin philosophe Galien (Ir siècle apr. J.-C.) mais qui, depuis la fin du XIX siècle, est attribué au philosophe néoplatonicien Porphyre (III siècle apr. J.-C.). En voici une admirable traduction commentée, précédée d'une riche introduction.

P. 7-9, un avant-propos de L. Brisson souligne le caractère collectif de ce livre et donne les références des traductions antérieures.

P. 13-17 sont exposés l'argument et le plan du traité. D’emblée, le texte est mis en rapport avec son arrière-plan médical et philosophique, soit essentiellement Hippocrate, Aristote et Platon, dont les théories embryologiques sont examinées par la suite.

P. 19-30, M.-H. Congourdeau présente une étude sur « L'embryologie dans le corpus hippocratique $\gg\left(V^{c}-I^{c}\right.$ siècle av. J.-C.). L'exposé, synthétique et biochronologique, examine successivement les sources de la connaissance de l'embryon, puis les questions de la semence, de la conception, du développement de l'embryon, de la formation, de la nourriture, de la respiration, des jumeaux, de la gestation, des anomalies, des accidents, enfin de l'accouchement. Les notes en bas de page, qui se contentent généralement de donner les références des textes cités, examinent parfois des questions très délicates, par exemple le caractère spéculatif de l'embryologic ou la traduction du terme meros, rendu par cellule ou parcelle.

P. 31-46, L. Brisson propose une étude sur « Le vivant, sa reproduction et sa nutrition selon Platon $\gg\left(\mathrm{IV}^{\complement}\right.$ siècle av. J.-C.). C'est surtout le Timée qui est étudié ici, avec quelques références aux Lois et au Phèdre. La définition platonicienne du vivant donne lieu à une théorie complexe, fondée notamment sur la distinction entre l'âme (psyche) et l'intellect (nous). La croyance en la métensomatose, croisée avec le phénomène de la reproduction, pose in fine la question du statut des plantes. Pour les passages les plus difficiles, l'article propose des traductions explicites, doublées de mises au point très claires, notamment en conclusion quand est analysée la justification platonicienne du végétarisme.

P. 47-67, G. Aubry étudie « La doctrine aristotélicienne de l'embryon et sa réinterprétation par Porphyre $\gg$. Les traités aristotéliciens concernés sont le $D e$ generatione animalium, le De anima et la Physique. L'article étudie rigoureusement la réception d'Aristote (IV siècle av. J.-C.) par Porphyre. Il s'agit de comprendre le lien que Porphyre établit entre son embryologie et la notion aristotélicienne d'enpuissance (dynamei). Porphyre pense que l'embryon reçoit l'âme de l'extérieur, au lieu de procéder à son développement de l'intérieur. En recourant aux notions 